\title{
Acrosomal status and mitochondrial activity of human spermatozoa vitrified with sucrose
}

\author{
E Isachenko, V Isachenko, J M Weiss, R Kreienberg, I I Katkov ${ }^{1}$, M Schulz ${ }^{3}$, A G-M I Lulat ${ }^{2}$, \\ M J Risopatrón ${ }^{3}$ and R Sánchez ${ }^{3}$ \\ Department of Obstetrics and Gynaecology, University Women's Hospital UIm, Pritwitzstrasse 43, 89075 Ulm, \\ Germany, ${ }^{1}$ Stem Cell Center, University of California at San Diego, La Jolla, California 92037, USA, ${ }^{2}$ CReATe Cord \\ Blood Bank, Toronto, Ontario, M5G 1N8, Canada and ${ }^{3}$ Department of Basic Sciences, Center of Biotechnology in \\ Reproduction, La Frontera University, Temuco, Chile
}

Correspondence should be addressed to E Isachenko; Email: e.isachenko@yahoo.de

\begin{abstract}
This study investigates the ability of sucrose to protect spermatozoa against mitochondrial damage, artificial cryoinduction of capacitation, and acrosome reaction. Spermatozoa were isolated using the swim-up procedure performed using three different media: (a) human tubal fluid (HTF, control) medium; (b) HTF with 1\% human serum albumin (HSA); and (c) HTF with $1 \%$ HSA and 0.25 M sucrose. From each group, $30 \mu \mathrm{l}$ suspensions of cells were dropped directly into liquid nitrogen and stored for at least $24 \mathrm{~h}$. Cells were thawed by quickly submerging the spheres in HTF with $1 \%$ HSA at $37^{\circ} \mathrm{C}$ with gentle agitation. Sperm motility, viability, mitochondrial membrane potential integrity, spontaneous capacitation, and acrosome reaction were investigated. Sperm viability, acrosome reaction, and capacitation were detected using the double fluorescence chlortetracycline-Hoechst 33258 staining technique. Mitochondrial function was evaluated using a unique fluorescent cationic dye, 5,5',6,6'-tetrachloro-1-1' $\mathbf{5}^{\prime}, 3,3^{\prime}$-tetraethyl-benzamidazolocarbocyanin iodide, commonly known as JC-1. The number of progressively motile spermatozoa was significantly higher in the sucrose-supplemented medium group $(57.1 \pm 3.2 \%, P<0.05)$ when compared with controls $(19.4 \pm 1.9 \%)$. The combination of HSA and sucrose $(65.2 \pm 2.6 \%)$ has a stronger cryoprotective effect on the integrity of mitochondrial membrane potential $(P<0.05)$ compared with HSA alone $(32.6 \pm 4.7 \%)$. In conclusion, vitrification of human spermatozoa with non-permeable cryoprotectants such as HSA and sucrose can effectively cryopreserve the cells without significant loss of important physiological parameters.
\end{abstract}

Reproduction (2008) 136 167-173

\section{Introduction}

Conventional slow freezing techniques are currently widely used in the cryopreservation of spermatozoa for reproductive medicine purposes. Vitrification is an alternative method that can also be applied to achieve the same purpose. The earliest information on vitrification comes from as far back as 1937. The technique arose as an alternative to conventional slow freezing, in order to avoid crystallization, which is incompatible with living systems (Luyet 1937). Successful vitrification of spermatozoa (Luyet \& Hoddap 1938) further supported Luyet's earlier work. However, subsequent attempts to vitrify mammalian spermatozoa using vitrification resulted in low or null survival because critical speeds of cooling were unachievable at that time. As a result, vitrification was not adopted as an alternative cryopreservation procedure at that time. In more recent times, vitrification has been applied successfully in the preservation of mouse embryos (Rall \& Fahy 1985).
However, it has not been possible to successfully apply the same technique to spermatozoa because of the use of high concentrations of permeable cryoprotectants and the osmotic and cytotoxic effects that they produce (Gilmore et al. 1997). Reduction in the use of some permeable and osmotic active non-permeable cryoprotectants has been suggested as an alternative (Nawroth et al. 2002). Isachenko et al. (2005) suggested four different vitrification techniques for spermatozoa. More recently, the use of carbohydrate supplements (glucose, sucrose, and trehalose) for cryopreservation by direct plunging into liquid nitrogen (LN) of human spermatozoa has also been studied (Schulz et al. 2006). It was noted that statistically the best post-warming spermatozoa motility and viability were obtained with $0.25 \mathrm{M}$ sucrose. The aim of this study was to investigate the ability of sucrose to protect spermatozoa against artificial cryoinduction of capacitation, acrosome reaction, and mitochondrial damage due to vitrification. 


\section{Results}

As a control, before vitrification, we tested the influence of medium (human tubal fluid, HTF), with and without the supplements (human serum albumin (HSA), sucrose), on the following parameters: spermatozoa motility, viability, capacitation, acrosome reaction, and influence on mitochondrial membrane potential stability before cryopreservation. It was established that HTF medium alone or in combination with HSA or sucrose does not have any significant negative influence on tested parameters in fresh sperm prior to cryopreservation. However, the viability of spermatozoa after $24 \mathrm{~h}$ culture in HTF medium alone was statistically lower than in HTF medium supplemented with HSA ( $P<0.05$; Fig. 1$)$.

The cryoprotective effect of HTF alone or in combination with HSA or HSA + sucrose on motility and viability of vitrified spermatozoa is illustrated in Fig. 2A. The data show that HTF alone (control group) has a low protective effect on spermatozoa motility $(19.4 \pm 1.9 \%$, $P<0.05)$. Supplementation of HTF medium by HSA only or by $\mathrm{HSA}+$ sucrose dramatically increases $(P<0.05)$ the motility rate $(45 \pm 3.7 \%$ and $57.1 \pm 3.2 \%$ respectively). The motility of warmed spermatozoa after $24 \mathrm{~h}$ culture shows a dramatic motility reduction for spermatozoa vitrified with HTF medium alone $(2.5 \pm 0.8 \%, P<0.05)$. The supplementation of HTF medium with HSA improved the survival of spermatozoa after $24 \mathrm{~h}$ culture $(21 \pm 3.1 \% ; P<0.05)$. However, the supplementing of HTF medium both with HSA and sucrose improved the survival of spermatozoa after $24 \mathrm{~h}$ culture $(32 \pm 5.9 \%, P<0.05)$ compared with the other two tested media (Fig. 2A). HTF alone or in combination with HSA or HSA + sucrose does not significantly affect the viability of spermatozoa in these vitrification experiments (Fig. 2A).

The percentage of capacitated and acrosome-reacted spermatozoa after vitrification in HTF alone or in combination with HSA or HSA+sucrose did not show any statistical difference between the groups (Fig. 2B, $P>0.1$ ).

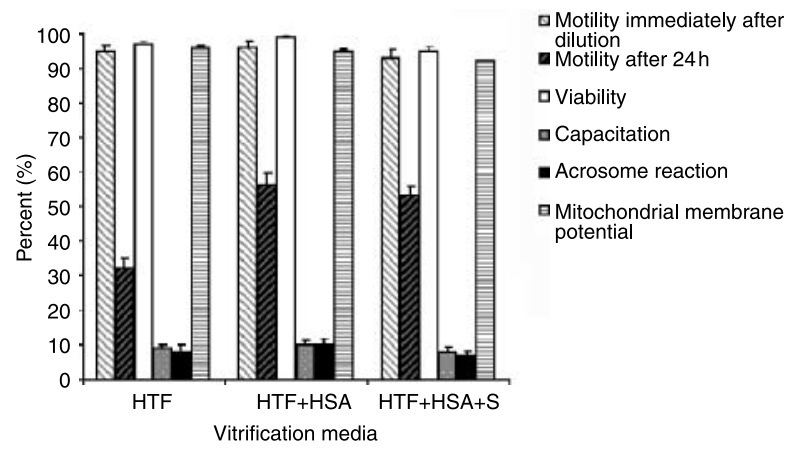

Figure 1 Influence of HTF alone or in combination with HSA or $\mathrm{HSA}+$ sucrose on motility, viability, activation of capacitation, induction of acrosome reaction, and mitochondrial membrane integrity before cryopreservation. No statistical differences were found between respective value of compared groups $(P>0.1)$ except the motility after $24 \mathrm{~h}$ in vitro culture $(P<0.05)$.
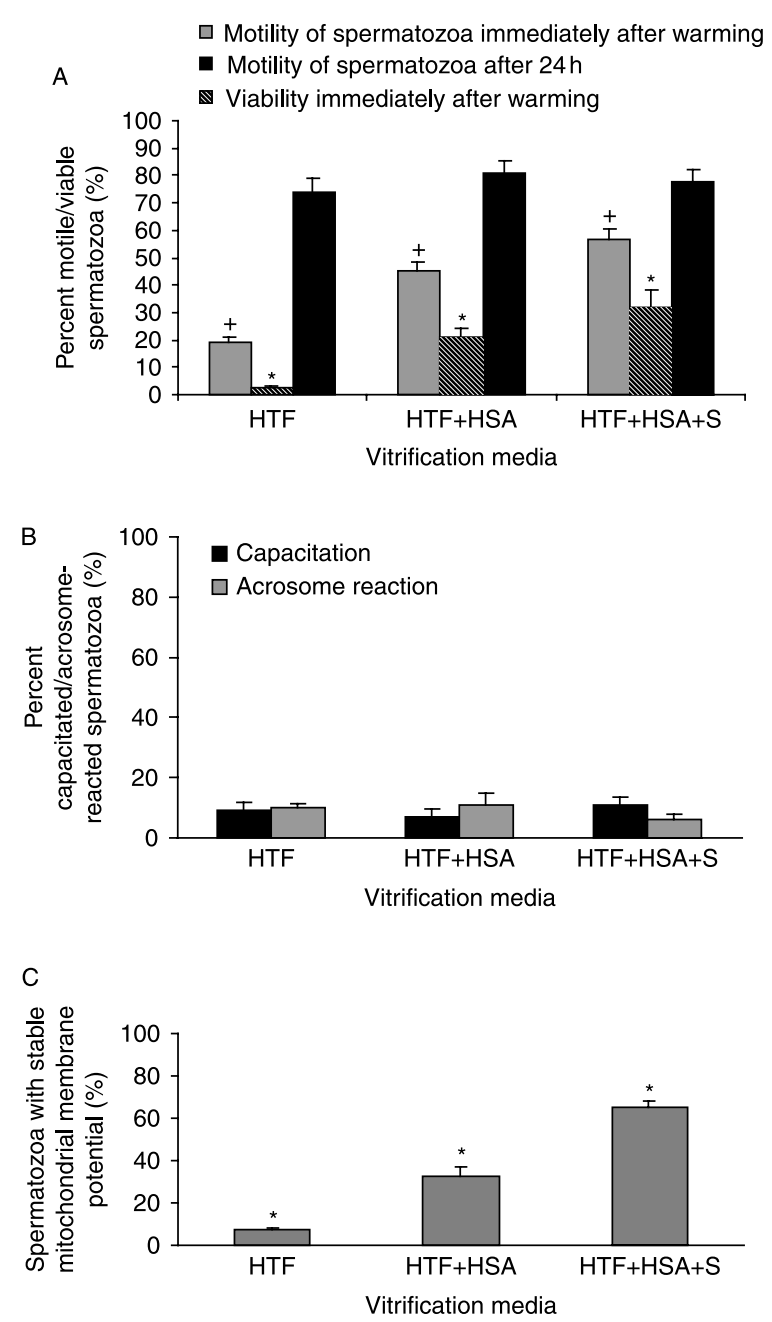

Figure 2 Cryoprotective effect of HTF alone or in combination with HSA or HSA + sucrose on (A) motility and viability of vitrified spermatozoa, (B) activation of capacitation and induction of acrosome reaction of vitrified spermatozoa and, (C) mitochondrial membrane integrity of vitrified spermatozoa. Asterisks denote statistical differences between respective value of compared groups $(P<0.05)$.

The cryoprotective effect of HTF alone or in combination with HSA or HSA+sucrose on the mitochondrial membrane is shown in Fig. 2C. It was observed that the HTF alone (control) has no cryoprotective effect $(7.7 \pm 0.7 \%, P>0.1)$. However, HTF in combination with HSA or HSA + sucrose does have a cryoprotective effect. The addition of HSA + sucrose to the HTF is more effective than the addition of HSA only $(65.2 \pm 2.6 \%$ and $32.6 \pm 4.7 \%$ respectively; $P<0.05)$.

\section{Discussion}

Since the time of discovery of the beneficial effects of glycerol and non-permeable cryoprotectants, including sucrose, on plant cryostability (Maksimov 1913), and the subsequent demonstration of the positive effect of glycerol on animal sperm cryoprotection (Bernschtein 
\& Petropavlovski 1937), it has been become clear that the effectiveness of a cryotechnique is associated with permeable and non-permeable cryoprotectants and can be revealed only with a low cooling rate (Gao et al. 1997). At present, in the management of human male infertility, the cryopreservation of sperm has become a routine technique. However, important properties of permeable cryoprotectants such as permeability and toxicity, which play a leading role in cryopreservation, are inseparably linked with osmotic damage that accompanies cell saturation before cooling and removal after thawing. It is also well known that the nonpermeable cryoprotectants, especially those used in the freezing of sperm from many mammalian species, play a supporting role and in most cases cannot protect the cells without the permeable cryoprotectants.

Conventional freezing techniques have been well investigated and it has been shown that these techniques cause extensive physical-chemical damage of extracellular and intracellular sperm membranes due to changes in lipid phase transition and/or increase in lipid peroxidation during saturation with cryoprotectants, freezing, or after thawing (Mossad et al. 1994). Lipid peroxidation leads to a decrease in sperm velocity, motility (Mossad et al. 1994), morphology, viability, and mitochondrial activities (O'Connell et al. 2002).

Vitrification on the other hand has its own peculiarities. The decisive factor in successful cryopreservation is the incompatibility of intracellular ice crystallization with living systems. To eliminate ice formation and create a glass-like (vitreous) state instead, it is necessary to cool small liquid suspensions or just water at ultra-high speeds of freezing (Luyet 1937). The physical definition of vitrification is the solidification of a solution at low temperature not by ice crystallization, but by extreme elevation in viscosity during cooling.

Achieving the vitrification conditions for the cryopreservation of oocytes or embryos without cryoprotectants is impossible at present, due to difficulties in achieving very high cooling rates. The addition of permeable cryoprotectants in high concentrations in combination with a 'slower' cooling rate from 2000 to $10000^{\circ} \mathrm{C} / \mathrm{min}$ by direct plunging of the vitrification solution into $\mathrm{LN}$ is an alternative. The protocols for vitrification are simple and demand only a few minutes of contact between the cells and the cryoprotectant solution before cooling. Unfortunately, this method of vitrification with the highly concentrated cryoprotectants is not suitable for mammalian spermatozoa due to the lethal effect of osmotic shock. The small droplet rapid freezing technique described in this paper achieves the desired vitrification. It is this drop-wise technique that is the key technical advance reported in this study.

In our earlier publications (Isachenko et al. 2004a, 2004b, 2005), we have achieved successful vitrification of spermatozoa without the use of permeable cryoprotectants. We were able to demonstrate a statistically higher motility of vitrified spermatozoa and their ability to fertilize in vitro compared with spermatozoa cryopreserved using conventional slow freezing. No statistical differences in parameters such as viability, recovery rate and percentage of morphologically normal spermatozoa with nondamaged DNA between vitrified and slow frozen cells were noted. However, it was observed that the number of spermatozoa with signs of acrosome reaction was statistically different $(P<0.001)$ when compared with fresh swim-up prepared spermatozoa. The importance of physiological parameters such as capacitation, acrosome reaction, and the integrity of cytoplasmic and mitochondrial membrane in fertilization are well known (Yanagimachi 1989, de Lamirande et al. 1997). These organelles are very cold sensitive (Drobnis et al. 1993, Holt 2000, O'Connel et al. 2002) and we have paid special attention to them in this investigation.

It is well known that mouse sperm are very sensitive compared with sperm from other mammalian species. Several studies have shown that mouse sperm do not tolerate osmotic stress during addition and removal of cryoprotectants (Songsasen \& Leibo 1997), and oxidative and mechanical stresses during pipetting or centrifugation (Katkov et al. 1998, Katkov \& Mazur 1999). In this case, the addition of a permeable cryoprotectant by way of dialysis to mouse spermatozoa (Thompson et al. 2001) allowed better recovery of cryopreserved cells. The effectiveness of the protocol for cryopreservation of mouse spermatozoa with a non-permeable cryoprotectant (raffinose) only has been shown (Nakagata \& Takeshima 1993). As a rule, carbohydrates are used for sperm cryopreservation to compensate for the decrease in osmotic pressure caused by the permeable cryoprotectant glycerol, which works as an additional dissolvent and has the ability to decrease the medium's osmotic pressure (Jakobsen 1956). It is suggested that raffinose plays the role of a membrane stabilizing and dehydrating agent (Wakayama et al. 1998). Koshimoto \& Mazur (2002) investigated three different sugars, i.e. monosaccharide glucose, disaccharide sucrose, and trisaccharide raffinose, and showed that protection against freezing/thawing injuries does not depend on the kind of sugar itself, but more on sugar's mass concentration than on its molar concentration, thus explaining mechanical implications. Based on this evidence, we decided to investigate the contribution of the disaccharide sucrose on sperm cryoprotection during cooling/warming. It is already known that human spermatozoa contain large amounts of proteins, sugars, and other components that make the intracellular matrix highly viscous and compartmentalized and may act as natural cryoprotectants. Since human spermatozoa are osmotically less fragile than mouse spermatozoa (Karlsson \& Cravalho 1994), they can be successfully frozen in the absence of permeable cryoprotectants, using protein- and sugar-rich extracellular nonpermeable cryoprotectants (Koshimoto et al. 2000). These findings (Karlsson \& Cravalho 1994, Koshimoto et al. 2000) helped us decide to investigate the ability of sucrose 
to prevent the artificial induction of capacitation, acrosome reaction, and support of mitochondrial stability during vitrification/warming.

Previously, others have suggested that cryopreservation of spermatozoa does not affect capacitation and the acrosome reaction (Esteves et al. 1998). According to their data, the acrosome reaction after cryopreservation may involve some complex mechanisms rather than a physiological change induced by capacitation. The results of our investigation corresponded to the data of that research group (Esteves et al. 1998) and showed a statistical difference between numbers of capacitated and acrosomereacted spermatozoa of control and experimental groups.

To detect mitochondrial competence, we used the probe JC-1, which is commonly used for the early detection of the initiation of cellular apoptosis. However, mature healthy human ejaculated spermatozoa are incapable of undergoing apoptosis (Lachaud et al. 2004); they lack the necessary assemblage of enzymes and signaling pathways possessed by cells capable of undergoing this process and the lack of orange-red fluorescence due to the binding of this cationic probe by the sperm mitochondria implies only the loss of energy conservation capacity. The mitochondria are most probably uncoupled rather than membrane damaged, but uncoupled mitochondria are evidence of unhealthy sperm and so this determination is useful to document post-thaw sperm survival by determination of mitochondrial activity. It has previously been demonstrated that mitochondrial activity and viability are equally susceptible to cryopreservation-induced damage (Meseguer et al. 2004). It has been suggested, however, that currently used conventional freezing techniques for human sperm cryopreservation are good enough to preserve all these structures $\left(\mathrm{O}^{\prime}\right.$ Connell et al. 2002, Meseguer et al. 2004).

In this study, we have shown that the inclusion of sucrose in combination with HSA in the vitrification medium has a visible cryoprotective effect on mitochondrial membrane integrity and provided significant protection to about $65 \%$ of spermatozoa compared with the control $(P<0.05)$. In the group with HSA alone, 33\% of spermatozoa showed intact mitochondrial membranes. The same positive effect of combination of these non-permeable cryoprotectants on spermatozoa motility after warming was also shown $(P<0.05)$.

In conclusion, we can infer that the drop-wise technique of sperm vitrification is a key technical advance, which allows the application of a mixture of non-permeable cryoprotectants: HSA and sucrose.

\section{Materials and Methods}

\section{Samples}

This study was approved by the appropriate University Review Boards. Except where otherwise stated, all chemicals were obtained from Sigma (Sigma Chemical Co).
After informed consent, ejaculates were obtained from 23 patients by masturbation after at least $48 \mathrm{~h}$ of sexual abstinence. All specimens had at least 20 million spermato$\mathrm{zoa} / \mathrm{ml}, 50 \%$ progressively motile, and $15-30 \%$ morphologically normal spermatozoa. Semen analysis was performed according to the published guidelines of the World Health Organization (1999).

All investigations were carried out on spermatozoa prepared by using the swim-up technique, as it allows for the selection of the most active, viable and morphologically normal fraction of ejaculated spermatozoa (Esteves et al. 2000). HTF (Quinn et al. 1985) medium was the basic medium used in sperm preparation and served as a control in the experiments.

Each ejaculate was divided into three equal parts. Swim-up for the first group was performed using HTF (control) medium and HTF with $1 \%$ HSA for the other two groups. After swim-up, the supernatants were centrifuged at $400 \mathrm{~g}$ for $5 \mathrm{~min}$ and diluted with the corresponding medium to achieve concentrations of $0.66 \times 10^{6} \mathrm{spermatozoa} / \mathrm{ml}$. The diluted suspensions of spermatozoa were again diluted (1:1) with the following media: the first part with pure HTF (control), the second part with HTF+ HSA, and the third part with $0.5 \mathrm{M}$ sucrose solution ( $0.25 \mathrm{M}$ end concentration). The $0.5 \mathrm{M}$ sucrose was prepared in bi-distillate water with HSA and after filtration with $0.22 \mu \mathrm{m}$ filter was frozen until use. After dilution, all the cell suspensions were maintained at $37^{\circ} \mathrm{C}$ in $5 \% \mathrm{CO}_{2}$ for $5 \mathrm{~min}$ before the cooling procedure was performed.

\section{Cooling and warming}

Initially, we tested various combinations of volumes and spermatozoa concentrations to identify the best ratio for our study. Volumes of 10, 20, 30, and $50 \mu \mathrm{l}$ and concentrations of $0.01,0.05,0.1$, and $0.2 \times 10^{6}$ spermatozoa $/ \mathrm{ml}$ were evaluated for progressive motility after vitrification and thawing. The postthaw motility was significantly greater when spermatozoa were suspended in $30 \mu \mathrm{l}$ volumes and at a final concentration of $0.01 \times 10^{6} / \mathrm{ml}$.

Vitrification was carried out as illustrated in Fig. 3. Briefly, a foam box was loaded with LN into which, for $5 \mathrm{~cm}$, a metal strainer was immersed. The strainer allows for easy collection of the spheres. Using a micropipette (10-100 $\mu$ l micropipette, Eppendorf, Hamburg, Germany) held at an angle of $45^{\circ}$ and at a distance of $10 \mathrm{~cm}$ from the $\mathrm{LN}$ surface, $30 \mu \mathrm{l}$ aliquots of spermatozoa suspension were dropped directly into the $\mathrm{LN}$. Upon contact with the LN, a sphere immediately forms and floats to the surface. After about $4 \mathrm{~s}$, the sphere solidifies and falls to the bottom of the strainer. Once this process has taken place, the procedure is repeated to obtain more spheres. It is important to wait for the sphere to precipitate before adding another microdrop, because they can stick together and form a drop of greater volume. After solidification, the spheres can be easily collected with the use of a small spoon. The solid spheres were packaged into $1.8 \mathrm{ml}$ cryotubes (Nunc, Brand Products, Roskilde, Denmark) and stored for at least $24 \mathrm{~h}$ in LN before use. Warming was performed by quickly submerging spheres one by one (not more than five spheres) into $5 \mathrm{ml} \mathrm{HTF}$ with $1 \%$ HSA pre-warmed to $37^{\circ} \mathrm{C}$ accompanied by gentle vortexing for 5-10 s. The post-thaw sperm suspension was maintained at 


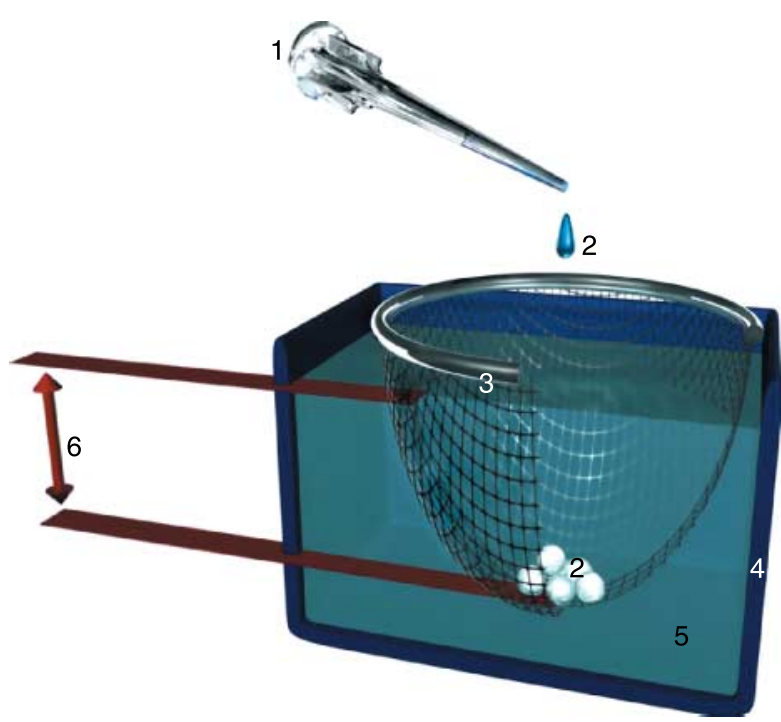

Figure 3 Scheme of the spermatozoa vitrification procedure. 1, single channel pipettes with adjustable volume $(30 \mu \mathrm{l}) ; 2$, spermatozoa suspension; 3, strainer; 4, foam box; 5, liquid nitrogen; and 6, distance between bottom of strainer and surface of $\mathrm{LN}$ (minimum $3 \mathrm{~cm}$ ).

$37^{\circ} \mathrm{C} / 5 \% \quad \mathrm{CO}_{2}$ for $10 \mathrm{~min}$ and then centrifuged at $380 \mathrm{~g}$ for $5 \mathrm{~min}$. The cell pellet was finally resuspended in $50 \mu \mathrm{l}$ HTF-HSA.

\section{Evaluation of sperm motility}

Sperm motility was assessed immediately after liquefaction, swim-up processing, dilution with the testing medium, warming of samples, and $24 \mathrm{~h}$ in vitro culture for evaluation of long-term spermatozoa survival. The Makler's chamber was used for all the motility studies. Motility was estimated under the light microscope using $\times 400$ magnification. Only spermatozoa with progressive motility (categories ' $a$ ' (rapid and regular forward progression) and ' $b$ ' (moderate, slow, or sluggish forward progression)) were assessed.

\section{Evaluation of viability, acrosome reaction, and capacitation}

The viable, acrosome-reacted, and capacitated spermatozoa were detected using the double fluorescence chlortetracycline (CTC)-Hoechst 33258 staining technique (Kay et al. 1994). In short, $100 \mu \mathrm{l}$ spermatozoa suspension were incubated with $1 \mu \mathrm{l}$ of $10 \mathrm{mg} / 100 \mu \mathrm{l} \mathrm{H}_{2} \mathrm{O}$ (Hoechst 33258) for $5 \mathrm{~min}$ at room temperature (RT) in the dark. After this, $1 \mathrm{ml}$ of $2 \%$ polyvinylpyrrolidone solution was added to the spermatozoa suspension and centrifuged at $400 \mathrm{~g}$ for $5 \mathrm{~min}$. The pellet was resuspended in $1 \mathrm{ml}$ PBS and centrifuged again at $400 \mathrm{~g}$ for $5 \mathrm{~min}$. After discarding the supernatant, the pellet was gently agitated and a $5 \mu \mathrm{l}$ suspension was placed in the center of a slide. Next, $5 \mu$ l of $1 \mathrm{mM} \mathrm{CTC}$ in $20 \mathrm{mM}$ Tris ( $\mathrm{pH}$ 7.8) solution were added to the droplet on the slide and incubated for $30 \mathrm{~s}$. The cells were fixed with 1:1 ratio of $25 \%$ glutaraldehyde in $1 \mathrm{M}$ Tris buffer. To retard the photobleaching during the fluorescence procedure, $0.22 \mathrm{mM}$ DABCO was used.
The probes were stored in the dark at $4{ }^{\circ} \mathrm{C}$ until reading (maximum $48 \mathrm{~h}$ ). At least 200 spermatozoa were observed in each plate and three patterns were identified (Fig. 4): (A) a uniform fluorescence on the head of the spermatozoa (noncapacitated spermatozoa); (B) a band of fluorescence diminished in the post-acrosomal region and a relatively shining fluorescence in the acrosomal region (capacitated spermatozoa); and (C) a fluorescence in the complete head of the spermatozoa, except a tenuous band of fluorescence in the equatorial segment (acrosome-reacted spermatozoa). The slides were viewed using a Zeiss Axiolab Epifluorescence microscope that was equipped with an excitation/emission filter of 485/ $520 \mathrm{~nm}$ under $\times 400$ magnification. The nonviable spermatozoa were observed with the filter set 09 (450-490 nm). The dead spermatozoa displayed a pattern of blue fluorescence in the whole head (Fig. 4D).

\section{Mitochondrial activity evaluation}

To evaluate mitochondrial activity, we measured changes in the mitochondrial membrane potential $(M \Delta \Psi)$ using a unique fluorescent cationic dye, 5, 5',6,6'-tetrachloro-1-1',3,3'-tetraethyl-benzamidazolocarbocyanin iodide, commonly known as JC-1 (Smiley et al. 1991). This test was performed as per the manufacturer's instructions for Mitochondrial Permeability Detection Kit AK-116 (MIT-E- $\Psi$, BIOMOL International LP, Plymouth Meeting, PA, USA). The $M \Delta \Psi$ was determined after incubation of $0.5 \times 10^{6}$ spermatozoa with $2.5 \mu \mathrm{l} \mathrm{JC}-1$ solution for $15 \mathrm{~min}$ at $37{ }^{\circ} \mathrm{C}$ in $5 \% \mathrm{CO}_{2}$. After this, the cell suspension was resuspended in $750 \mu \mathrm{l} \mathrm{HTF}$ and centrifuged at $400 \mathrm{~g}$ for 5 min at RT. After the removal of supernatant, the spermatozoa were observed under the epifluorescence microscope (Axiolab drb, KT 450905, Zeiss) at $\times 400$ magnification. For MIT-E- $\Psi$ reagent, an excitation/emission filter of $488 / 490 \mathrm{~nm}$ was used. The monomeric dye structure emits at $527 \mathrm{~nm}$, whereas J-aggregates in healthy (non-damaged) mitochondria emit at $590 \mathrm{~nm}$. If the $M \Delta \Psi$ is intact, the JC-1 reagent will aggregate
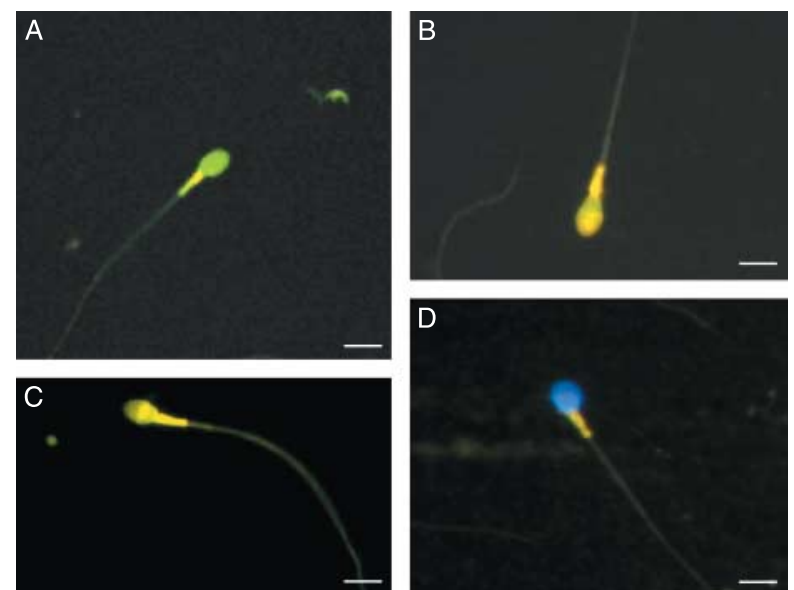

Figure 4 Example of (A) non-capacitated, (B) capacitated, (C) acrosomereacted, and (D) nonviable spermatozoa. The capacitated, acrosomereacted, and nonviable spermatozoa were detected using the double fluorescence chlortetracycline (CTC)-Hoechst 33258 staining technique. 

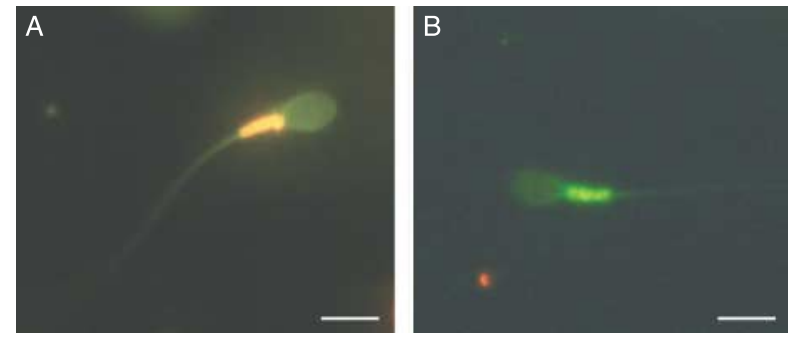

Figure 5 Example of (A) non-damaged and (B) damaged mitochondria. In non-damaged mitochondria, the $M \Delta \Psi$ is intact and the JC-1 reagent aggregate inside the non-damaged mitochondria and fluoresce red. In our case, the midpiece is yellow, as expected for the red fluorescence from JC-1 aggregates merging with the green fluorescence of JC-1 monomer dispersed throughout the cell plasma membrane. In damaged mitochondria, the $M \Delta \Psi$ is breaking down, and the JC-1 reagent dispersed through the entire cells and fluoresce green. The changes in the mitochondrial membrane potential $(M \Delta \Psi)$ were measured using a unique fluorescent cationic dye, 5, 5',6,6'-tetrachloro-1-1',3,3'-tetraethyl-benzamidazolocarbocyanin iodide, commonly known as JC-1.

inside the healthy non-damaged mitochondria and fluoresce red (Fig. 5A). In our case, the midpiece is yellow, as expected for the red fluorescence from JC-1 aggregates merging with the green fluorescence of JC-1 monomer dispersed throughout the cell plasma membrane. If the mitochondria are damaged, the $M \Delta \Psi$ will be breaking down and the JC-1 reagent will be seen dispersed though the entire cells and fluoresce green (Fig. 5B). A total of 200 cells were analyzed on each slide.

\section{Statistical analysis}

For the statistical analysis, we used the nonparametric ANOVA (Kruskal-Wallis) with a significance level of 0.05 and the test of multiple comparison of Dunn to establish differences between the groups.

\section{Declaration of interest}

The authors declare that there is no conflict of interest that would prejudice the impartiality of this scientific work.

\section{Funding}

This research did not receive any specific grant from any funding agency in the public, commercial or not-for-profit sector.

\section{Acknowledgements}

The authors thank Mr F Braun for preparation of the manuscript.

\section{References}

Bernschtein AD \& Petropavlovski VV 1937 Influence of non-electrolytes on viability of spermatozoa. Bulletin of Experimental Biology and Medicine III 21-25.
Drobnis EZ, Crowe LM, Berger T, Anchordoguy TJ, Overstreet JW \& Crowe JH 1993 Cold shock damage is due to lipid phase transitions in cell membranes: a demonstration using sperm as a model. Journal of Experimental Zoology 256 432-437.

Esteves SC, Sharma RK, Thomas AJ Jr \& Agarwal A 1998 Effect of in vitro incubation on spontaneous acrosome reaction in fresh and cryopreserved spermatozoa. International Journal of Fertility and Women's Medicine 43 235-242.

Esteves SC, Sharma RK, Thomas AJ \& Agarwal A 2000 Improvement in motion characteristics and acrosome status in cryopreserved human spermatozoa by swim-up processing before freezing. Human Reproduction 15 2173-2179.

Gao D, Mazur P \& Critser J 1997 Fundamental cryobiology of mammalian spermatozoa. In Reproductive Tissue Banking, 4 edn, pp 263-328. Eds AM Karow \& JK Critser. London: Academic Press.

Gilmore JA, Liu J, Gao DY \& Critser JK 1997 Determination of optimal cryoprotectants and procedures for their addition and removal from human spermatozoa. Human Reproduction 12 112-118.

Holt WV 2000 Fundamental aspects of sperm cryobiology: the importance of species and individual differences. Theriogenology 53 47-58.

Isachenko E, Isachenko V, Katkov II, Rahimi G, Schondorf T, Mallmann P, Dessole S \& Nawroth F 2004a DNA integrity and motility of human spermatozoa after standard slow freezing versus cryoprotectant-free vitrification. Human Reproduction 19 932-939.

Isachenko V, Isachenko E, Katkov II, Montag M, Dessole S, Nawroth F \& van der Ven H 2004b Cryoprotectant-free cryopreservation of human spermatozoa by vitrification and freezing in vapor: effect on motility, DNA integrity, and fertilization ability. Biology of Reproduction 71 1167-1173.

Isachenko V, Isachenko E, Montag M, Zaeva V, Krivokharchenko A, Nawroth F, Dessole S, Katkov I \& van der Ven H 2005 Clean technique for cryoprotectant - free vitrification of human spermatozoa. Reproductive Biomedicine Online 10 350-354.

Jakobsen FK 1956 Forsog reed dybfrysning af tyresaed. In Beretning fra Forsogslaboratoriet, p 292. Kobenhavn.

Karlsson JOM \& Cravalho EG 1994 A model of diffusion-limited ice growth inside biological cells during freezing. Journal of Applied Physics 75 4442-4455.

Katkov II \& Mazur P 1999 Factors affecting yield and survival of cell when suspensions are subjected to centrifugation. Influence of centrifugal acceleration, time of centrifugation, and length of the suspension column in quasi-homogeneous centrifugal fields. Cell Biochemistry and Biophysics 31 231-245.

Katkov II, Katkova N, Critser JK \& Mazur P 1998 Mouse spermatozoa in high concentration of glycerol: chemical toxicity vs osmotic shock at normal and reduced oxygen concentrations. Cryobiology 37 325-338.

Kay VJ, Coutts JR \& Robertson L 1994 Effects of pentoxifylline and progesterone on human sperm capacitation and acrosome reaction. Human Reproduction 9 2318-2323.

Koshimoto C \& Mazur P 2002 The effect of the osmolality of sugarcontaining media, the type of sugar, and the mass and molar concentration of sugar on the survival of froze-thawed mouse sperm. Cryobiology 45 80-90.

Koshimoto C, Gamliel E \& Mazur P 2000 Effect of osmolality and oxygen tension on the survival of mouse sperm frozen to various temperatures in various concentrations of glycerol and raffinose. Cryobiology $\mathbf{4 1}$ 204-231.

Lachaud C, Tesarik J, Canadas ML \& Mendoza C 2004 Apoptosis and necrosis in human ejaculated spermatozoa. Human Reproduction 19 607-610.

de Lamirande E, Leclerc P \& Gagnon C 1997 Capatitation as a regulatory event that primes spermatozoa for the acrosome reaction and fertilization. Molecular Human Reproduction 3 175-194.

Luyet BJ 1937 The vitrification of organic colloids and of protoplasm. Biodynamica 1 1-14.

Luyet BJ \& Hoddap A 1938 Revival of frog's spermatozoa vitrified in liquid air. Proceedings of the Meeting of Society for Experimental Biology 39 433-434.

Maksimov NA 1913 On destroying by frost and cold-resistant of plants. Izvestija Sibirskogo Lesnogo Instituta 25 5-10. 
Meseguer M, Garrido N, Martinez-Conejero JA, Simon C, Pellicer A \& Remohi J 2004 Role of cholesterol, calcium, and mitochondrial activity in the susceptibility for cryodamage after a cycle of freezing and thawing. Fertility and Sterility 82 514-515.

Mossad H, Morshedi M, Torner JP \& Oehninger S 1994 Impact of cryopreservation on spermatozoa from infertile men - implication for artificial insemination. Archives of Andrology 33 51-57.

Nakagata N \& Takeshima T 1993 Cryopreservation of mouse spermatozoa from inbred and F1 hybrid strains. Jikken Dobutsu 42 317-320.

Nawroth F, Isachenko V, Dessole S, Rahimi G, Farina M, Vargiu N \& Mallmann PI 2002 Vitrification of human spermatozoa without cryoprotectants. Cryo Letters 23 93-102.

O'Connell M, McClure N \& Lewis SEM 2002 The effects of cryopreservation on sperm morphology, motility and mitochondrial function. Human Reproduction 17 704-709.

Quinn P, Warnes GM, Kerin JF \& Kirby C 1985 Culture factors affecting the success rate of in vitro fertilization and embryo transfer. Annals of the New York Academy of Sciences 442 195-204.

Rall WF \& Fahy GM 1985 Ice-free cryopreservation of mouse embryos at $-196^{\circ} \mathrm{C}$ by vitrification. Nature 313 573-575.

Schulz M, Muñoz M, Risopatrón J \& Sánchez R 2006 Cryopreservation of human spermatozoa by vitrification. International Journal of Morphology 2431.

Smiley ST, Reers M, Motolla-Hartshorn C, Lin M, Chen A, Smith TW, Steele GD \& Chen LB 1991 Intracellular heterogeneity in mitochondrial membrane potentials revealed by a J-aggregate-forming lipophilic cation JC-1. PNAS 88 3671-3675.
Songsasen N \& Leibo S 1997 Cryopreservation of mouse spermatozoa: II relationship between survival after cryopreservation and osmotic tolerance of spermatozoa from three strains of mice. Cryobiology 35 255-269.

Thompson KA, Richa J, Liebhaber SA \& Storey B 2001 Dialysis addition of trehalose/glycerol cryoprotectant allows recovery of cryopreserved mouse spermatozoa with satisfactory fertilizing ability as assessed by yield of live young. Journal of Andrology 22 339-344.

Wakayama T, Whittinhgam DG \& Yanagimachi R 1998 Production of normal offspring from mouse oocytes injected with spermatozoa cryopreserved with or without cryoprotection. Journal of Reproduction and Fertility 112 11-17.

World Health Organization 1999 Laboratory Manual for the Examination of Human Semen-Cervical Mucus Interaction, 4 Cambridge: Cambridge University Press.

Yanagimachi R 1989 Sperm capacitation and gamete interaction. Journal of Reproduction and Fertility 38 27-33.

Received 23 June 2007

First decision 27 July 2007

Revised manuscript received 5 April 2008

Accepted 14 May 2008 